\title{
FEATURES OF SIMULATION OF CHARACTERISTICS OF THERMOMETRIC MATERIAL $\mathrm{Lu}_{1-x} \mathrm{Zr}_{x} \mathrm{NiSb}$
}

\author{
Volodymyr Krayovskyy, Dr. Sc., Prof., Volodymyr Pashkevych, Ph. Dr., As.-Prof., \\ Andriy Horpenuk, Ph. Dr., As.-Prof., Volodymyr Romaka, Dr. Sc., Prof., \\ Lviv Polytechnic National University, Ukraine \\ Yuriy Stadnyk, Ph. Dr., Senior Scientist, Lyubov Romaka, Ph. Dr., Senior Scientist, \\ Andriy Horyn, Ph. Dr., Senior Research, \\ Ivan Franko National University of Lviv, Ukraine \\ Vitaliy Romaka, Dr. Sc., Prof., \\ Technische Universität Dresden, Dresden, Germany \\ e-mail:vkrayovskyy@ukr.net
}

\begin{abstract}
The results of modeling the thermometric characteristics of the semiconductor solid solution $\mathrm{Lu}_{1-x} \mathrm{Zr}_{x} \mathrm{NiSb}$, which is a promising thermometric material for the manufacture of sensitive elements of thermoelectric and electro resistive thermocouples, are presented. Modeling of the electronic structure of $\mathrm{Lu}_{1-x} \mathrm{Zr}_{x} \mathrm{NiSb}$ was performed by the Korringa-Kohn-Rostoker (KKR) method in the approximation of coherent potential and local density and by the full-potential method of linearized plane waves (FLAPW). KKR simulations were performed using the AkaiKKR software package in the local density approximation for the exchange-correlation potential with parameterization Moruzzi, Janak, Williams in the semi-relativistic one taking into account the spin-orbit interaction. The implementation of the method in the Elk software package was used to perform FLAPW calculations. To check the limits of the existence of the thermometric material $\mathrm{Lu}_{1-x} \mathrm{Zr}_{x} \mathrm{NiSb}$, both methods were used to calculate the change in the values of the period of the unit cell $a(x)$ in the range $x=0-1.0$. It is shown that there is an agreement between the change in the values of $a(x) \mathrm{Lu}_{1-x} \mathrm{Zr}_{x} \mathrm{NiSb}$ calculated by the FLAPW method and the results of experimental studies. The obtained result indicates higher accuracy of modeling of structural parameters $\mathrm{Lu}_{1-x} \mathrm{Zr}_{x} \mathrm{NiSb}$ by the FLAPW method in comparison with the KKR method. To study the possibility of obtaining thermometric material $\mathrm{Lu}_{1-x} \mathrm{Zr}_{x} \mathrm{NiSb}$ and to establish the limits of its existence in the form of a continuous solid solution, modeling of thermodynamic characteristics in the approximation of harmonic oscillations of atoms within the theory of DFT density functional for a hypothetical solid solution $\mathrm{Lu}_{1-x} \mathrm{Zr}_{x} \mathrm{NiSb}, x=0-1.0$. The change in the values of the enthalpy of mixing $\Delta H$ and the total energy $E \mathrm{Lu}_{1-x} \mathrm{Zr}_{x} \mathrm{NiSb}, x=0-1.0$, allows us to state that the thermometric material exists in the form of a solid substitution solution in the concentration range $x=0-<0.20$. At higher concentrations of impurity $\mathrm{Zr}$ atoms, $x>0.20$, stratification occurs (spinoidal phase decay) and thermometric material does not exist.

To understand the mechanisms of electrical conductivity of the thermometric material $\mathrm{Lu}_{1-x} \mathrm{Zr}_{x} \mathrm{NiSb}$, the methods of entry of impurity $\mathrm{Zr}$ atoms into the matrix of the basic semiconductor $p$-LuNiSb and their occupation of different crystallographic positions, as well as the presence of vacancies in them, were investigated. For this purpose, its electronic structure was modeled for different variants of the spatial arrangement of atoms and the presence of vacancies in crystallographic positions. It is shown that the most acceptable results of experimental studies are the model of the electronic structure of $p$-LuNiSb, which assumes the presence of vacancies in the crystallographic positions of $4 a \mathrm{Lu}$ atoms $(\sim 0.005)$ and $4 c \mathrm{Ni}$ atoms $(\sim 0.04)$. In this model of the spatial arrangement of atoms and the presence of vacancies at positions $4 a$ and $4 c$, the LuNiSb compound is a semiconductor of the hole-type conductivity, in which the Fermi level $\varepsilon_{F}$ is located near the level of the valence band $\varepsilon_{V}$.

The kinetic characteristics of the semiconductor thermometric material $\mathrm{Lu}_{1-x} \mathrm{Zr}_{x} \mathrm{NiSb}$, in particular, the temperature dependences of the resistivity $\rho(T, x)$ and the thermopower coefficient $\alpha(T, x)$ are modeled. It is established that at the lowest concentrations of impurity atoms $\mathrm{Zr}$ the Fermi level $\varepsilon_{F} \mathrm{Lu}_{1-x} \mathrm{Zr}_{x} \mathrm{NiSb}$ passes from the bandgap to the conduction band $\varepsilon_{C}$. This is indicated by the negative values of the thermopower coefficient $\alpha(T, x)$ and the metallic conductivity type $\mathrm{Lu}_{1-x} \mathrm{Zr}_{x} \mathrm{NiSb}$. This changes the type of main current carriers from holes to electrons.
\end{abstract}

Key words: Electric conductivity; Thermopower coefficient; Fermi level.

\section{Introduction}

Interest in thermometric materials based on $p$ $\mathrm{RNiSb}$ semiconductors (where $\mathrm{R}$ is a rare earth metal of the yttrium subgroup) is due to both high values of thermopower coefficient $\alpha(T)$ and electrical resistance $\rho(T)$, which makes these materials one of the most promising [1]. Studies of the influence of alloying impurities on the change of crystal and electronic structures, distribution of density of electronic states (DOS), kinetic, magnetic, and energy characteristics allowed us to identify features of electrical conductivity mechanisms and develop ways to optimize material parameters to obtain high resistance and thermopower. The process of optimizing the parameters of thermometric materials by doping the basic semiconductor with donor and/or acceptor impurities involves computer modeling of its characteristics. The choice of conditions for its synthesis, in particular, the concentration of impurity atoms and the temperature of homogenizing 
annealing, which determines its thermometric characteristics, depends on the correctness of the chosen method of modeling the characteristics of the material.

The study of structural, kinetic, and magnetic characteristics of RNiSb compounds $(R=\mathrm{Gd}, \mathrm{Tb}, \mathrm{Dy}, \mathrm{Yb}$, $\mathrm{Lu}$ ) found that the crystal structure of the compound $\mathrm{YbNi}_{0.9} \mathrm{Sb}$ is defective, and the compounds themselves are semiconductors of the hole-type conductivity [1]. Thus, in the crystal, there is a mechanism for generating structural defects of acceptor nature. However, as of now, there are no perfect models of the crystal and electronic structures of $\mathrm{RNiSb}$ that are adequate to the real state of the semiconductor material. The main reason for this state is the wrong choice of methods for modeling the characteristics, which do not fully take into account all the features of the material, in particular, the crystal structure to the density distribution of electronic states.

The results of modeling the structural, thermodynamic, energy, and kinetic characteristics of the thermometric material $\mathrm{Lu}_{1-x} \mathrm{Zr}_{x} \mathrm{NiSb}$ obtained by doping the basic semiconductor LuNiSb with $\mathrm{Zr}$ atoms by substituting $\mathrm{Lu}$ atoms $(4 a)$ are presented. The obtained results will allow us to clarify the spatial arrangement of atoms in the nodes of the unit cell, as well as to identify the mechanisms of electrical conductivity to determine the conditions for the synthesis of thermosensitive materials with maximum efficiency of thermal energy conversion into electricity.

\section{Disadvantages}

Studies of thermometric materials based on the basic semiconductor RNiSb [1] have established their high sensitivity to heat treatment (temperature and duration of annealing).

\section{Research Objective}

To establish the mechanism of formation of structural defects in the thermometric material $\mathrm{Lu}_{1-x} \mathrm{Zr}_{x} \mathrm{NiSb}$, which will permit to identify the mechanisms of electrical conductivity and to determine the conditions of their synthesis.

\section{Research Methods}

Calculations of the electronic structure, density distribution of electronic states (DOS), electron localization function (ELF), thermodynamic characteristics, as well as optimization of the crystal structure parameters of the $\mathrm{Lu}_{1-x} \mathrm{Zr}_{x} \mathrm{NiSb}$ thermometric material were performed using the Koringa-Kon-Rostoker method (hereinafter KKR) in the Coherent Potential Approximation (CPA) and Local Density Approximation (LDA) approximation, as well as the Full Potential Linearized Augmented Plane Waves (FLAPW) method. KKR simulations were performed using AkaiKKR software packages [2] in the local density approximation for the exchange-correlation potential with parameterization Moruzzi, Janak, Williams [3] (MJW) in the semi-relativistic consideration of the core level taking into account the spin-orbit interaction. The implementation of the method in the Elk software package was used for FLAPW calculations [4]. The simulation was performed for a $10 \times 10 \times 10 \mathrm{k}$-grid in the approximation of both the local density (LDA) and the generalized GGA gradient. The Brillouin zone was divided into $1000 \mathrm{k}$ points, which were used to calculate both the Bloch spectral function (band energy spectrum) and the density of electronic states. The width of the energy window was chosen to capture the semi-core states of $p$-elements. Visualization of volumetric data was performed using the program VESTA [5]. Topological analysis and interpretation of DOS and ELF were performed within the framework of Bader's theory.

\section{Modeling of Structural Characteristics of Thermometric Material Lu1-xZrxNiSb}

To check the existence of the boundaries of the thermometric material $\mathrm{Lu}_{1-x} \mathrm{Zr}_{x} \mathrm{NiSb}$, the change of the values of the period of the unit cell $a(x)$ was calculated using the software packages AkaiKKR [3] and Elk [4] (Fig. 1). The results of the calculation of the change in the values of $a(x) \mathrm{Lu}_{1-x} \mathrm{Zr}_{x} \mathrm{NiSb}$ by both methods show a decrease in the values of the cell period, which is quite logical since the atomic radius $\mathrm{Zr}\left(a_{\mathrm{Zr}}=0.160 \mathrm{~nm}\right)$ is smaller than that of $\mathrm{Lu}\left(a_{\mathrm{Lu}}=0.173 \mathrm{~nm}\right)$. From Fig. 1 also shows that the values of the unit cell period a of the LuNiSb compound obtained by simulation using the AkaiKKR method [2] are smaller than those obtained by using the method in the Elk software package [4]. At the same time, the simulation result for the hypothetical compound $\mathrm{ZrNiSb}$ (the other side of the solid solution $\mathrm{Lu}_{1-x} \mathrm{Zr}_{x} \mathrm{NiSb}$ at $x=1$ ) showed the opposite result: the values of period a of the compounds $\mathrm{ZrNiSb}$ obtained by the method AkaiKKR [2] are greater than those obtained by the method Elk [4-9] (Fig. 1). We can state that the course of the dependences $a(x) \operatorname{Lu}_{1-x} \mathrm{Zr}_{x} \mathrm{NiSb}$ obtained by different modeling methods follows different laws. In this case, the angles of inclination of the dependences $a(x) \mathrm{Lu}_{1-x} \mathrm{Zr}_{x} \mathrm{NiSb}$ are different. The obtained result indicates a higher accuracy of modeling the structural parameters of $\mathrm{Lu}_{1-x} \mathrm{Zr}_{x} \mathrm{NiSb}$, in particular, the period of the cell $a(x)$ by the FLAPW method in comparison with the KKR method.

The formation of the semiconductor thermometric material $\mathrm{Lu}_{1-x} \mathrm{Zr}_{x} \mathrm{NiSb}$ by substituting $\mathrm{Lu}$ atoms for $\mathrm{Zr}$ at position $4 a$ is accompanied by changes in both the crystal structure and the electron structure. After all, the introduction of $\mathrm{Zr}$ atoms $\left(4 d^{2} 5 s^{2}\right)$ into the structure of the LuNiSb compound by substituting $\mathrm{Lu}$ atoms at position $4 a\left(5 d^{1} 6 s^{2}\right)$ should generate structural defects of donor 
nature, since the $\mathrm{Zr}$ atom contains a larger number of $d$-electrons than the Lu atom. In the experiment, this should lead to a change in the type of main carriers of electric current from hole to electronic.

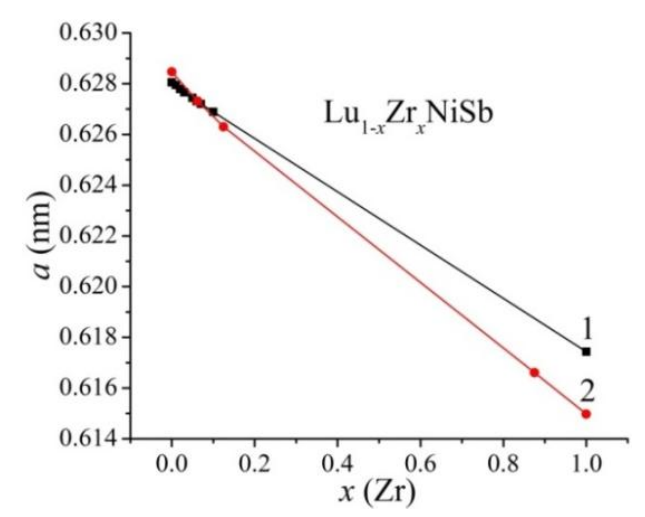

Fig. 1. The change in the values of the period of the unit cell a (x) of the thermometric material $\mathrm{Lu}_{1-x} \mathrm{Zr}_{x} \mathrm{NiSb}$ calculated by the methods AkaiKKR (1) and Elk (2)

\section{Modeling of Thermodynamic}

\section{Characteristics of $\mathrm{Lu}_{1-x} \mathrm{Zr}_{x} \mathrm{NiSb}$}

Based on the fact that there is no $\mathrm{ZrNiSb}$ compound with the structure of $\mathrm{MgAgAs}$, and hence a continuous solid solution ( $100 \%$ substitution in the structure of compounds of $\mathrm{Lu}$ atoms on $\mathrm{Zr}$ and vice versa), it is important to establish the possible existence of ther-

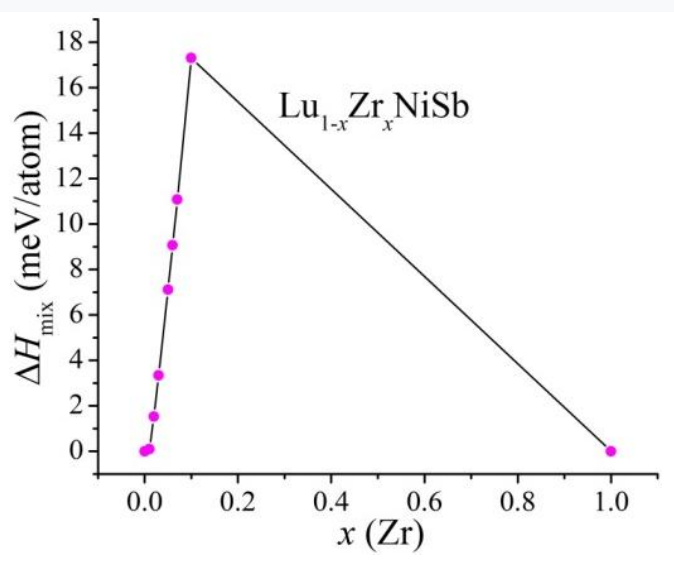

$a$ mometric material $\mathrm{Lu}_{1-x} \mathrm{Zr}_{x} \mathrm{NiSb}$ obtained by substitution in crystallography position $4 a$ of Lu atoms on $\mathrm{Zr}$. To study the possibility of obtaining thermometric material $\mathrm{Lu}_{1-}$ ${ }_{x} \mathrm{Zr}_{x} \mathrm{NiSb}$ and to establish the limits of its existence, modeling of thermodynamic characteristics in the approximation of harmonic oscillations of atoms within the theory of DFT density functional for a hypothetical solid solution $\mathrm{Lu}_{1-x} \mathrm{Zr}_{x} \mathrm{NiSb}, x=0-1.0$. The change in the values of the enthalpy of mixing $\Delta H$ (Fig. 2,a) and the total energy $\Delta E$ (Fig. 2, b) $\mathrm{Lu}_{1-x} \mathrm{Zr}_{x} \mathrm{NiSb}, x=0-1.0$, allows us to state that the thermometric material exists as a solid substitution solution in the concentration range $x=0-<0.20$. In this concentration region, the values of the enthalpy of mixing $\Delta H(x)$ and the total energy $\Delta E(x)$ increase, indicating the energy expediency of substituting $\mathrm{Lu}$ atoms for $\mathrm{Zr}$. However, at higher concentrations of impurity atoms $\mathrm{Zr}, x>0.20$, the dependences $\Delta H(x)$ and $\Delta E(x)$ decrease, which indicates the energy disadvantage of the formation of a solid substitution solution. Stratification occurs (spinoidal phase decay) and thermometric material does not exist.

Thus, the results of modeling the enthalpy of mixing $\Delta H$ and total energy $\Delta E \mathrm{Lu}_{1-x} \mathrm{Zr}_{x} \mathrm{NiSb}$ suggest that there is no unlimited solubility of $\mathrm{Zr}$ atoms in the structure of the basic semiconductor LuNiSb, and the concentration of impurity atoms $\mathrm{Zr}, x \approx 0.2$, is the limit of the existence of $Z$ occupy the crystallographic position $4 a$. At higher concentrations of $\mathrm{Zr}$ atoms, $x>0.20$, stratification occurs (spinoidal phase decay).

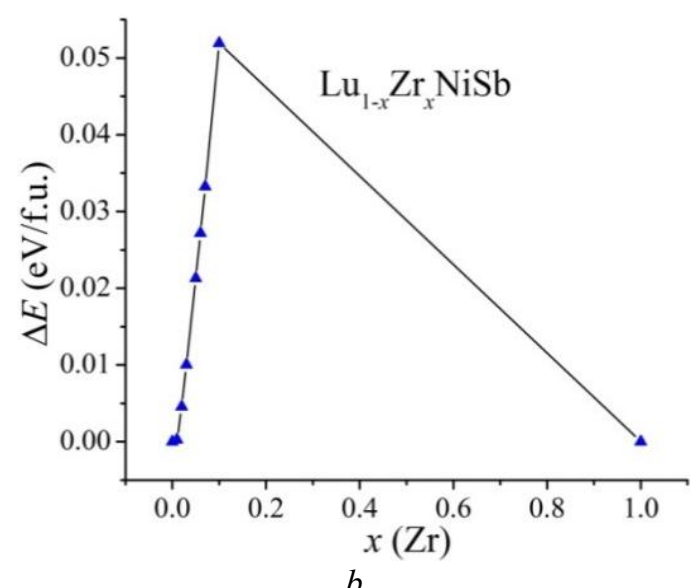

Fig. 2. Calculation by the Elk method of changing the values of the enthalpy of mixing $\Delta H$ (a) and the total energy $\Delta E$ (b) of the thermometric material $\mathrm{Lu}_{1-x} \mathrm{Zr}{ }_{x} \mathrm{NiSb}$

\section{LuNiSb}

\section{Modeling of Energy Characteristics of}

The first and most important step in modeling the electronic structure of the semiconductor thermometric material $\mathrm{Lu}_{1-x} \mathrm{Zr}_{x} \mathrm{NiSb}$, in particular, the behavior of the Fermi level $\varepsilon_{F}$, the bandgap $\varepsilon_{q}$ is to understand the features of the spatial location and electronic structure of the basic semiconductor $p$-LuNiSb. It is these features that determine how impurity atoms enter the semiconductor matrix, which determines the formation of structural defects of different nature and the appearance of corresponding energy levels in the bandgap. If we take as a basis an ordered model of the crystal structure of the compound LuNiSb, in which all crystallographic positions are occupied by atoms following the structural type of MgAgAs, then modeling of the electronic structure of LuNiSb shows that the 
compound is a semiconductor of electronic conductivity type (Fig. 3). Accordingly, the Fermi level $\varepsilon_{F}$ (dotted line) is located near the conduction band $\varepsilon_{C}$, which in the experiment will give negative values of the thermopower coefficient $\alpha(T)$ at all temperatures. However, this result of modeling the electronic structure of the $p$-LuNiSb semiconductor does not agree with the results of experimental studies [1], where positive values of the thermopower coefficient $\alpha(T)$ were obtained.

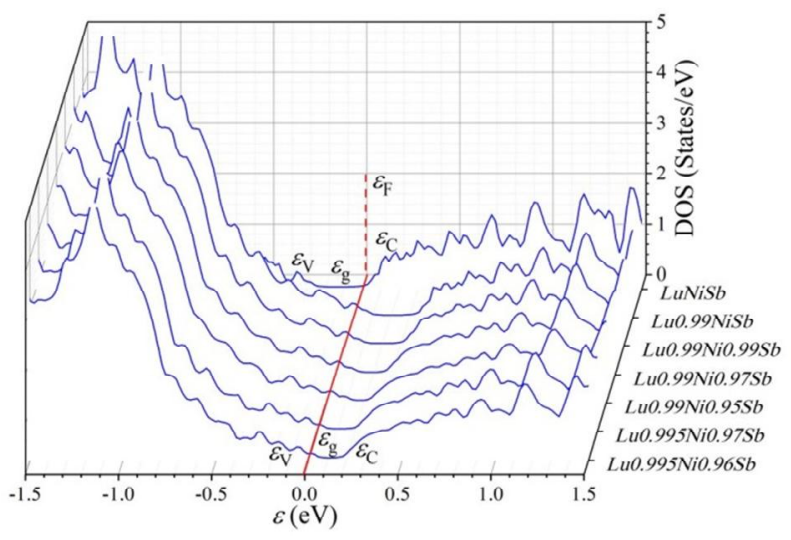

Fig. 3. Calculation of the density distribution of electronic states of DOS for different employment options of the crystallographic positions of the compound LuNiSb

The inconsistency of the results of experimental studies of the basic $p$-LuNiSb semiconductor and the modeling of the electronic structure for the ordered model of its crystal structure indicates its disorder. Thus, in the crystal structure of the compound LuNiSb, there is a partial occupation of atoms of foreign positions, and it is also possible to have vacancies in different crystallographic positions. After all, in the case of doping the base semiconductor with impurity atoms, the presence of vacancies and the occupation of crystallographic positions will determine the ways of formation of structural defects and energy levels in the bandgap $\varepsilon_{q}$. That is why it is important to establish the features of the crystal structure of the basic semiconductor $p$-LuNiSb. To establish the crystal structure of LuNiSb, as close as possible to its real state, which is reflected in agreement with the results of experimental measurements [1], the electronic structure of LuNiSb was modeled for different variants of the spatial arrangement of atoms and vacancies in different crystallographic positions. In Fig. 3, $a$ fragment of the results of modeling the density distribution of electronic states for an ordered model of the crystal structure (all atoms occupy their positions) of the LuNiSb compound is shown, but the crystallographic positions $4 a$ of $\mathrm{Lu}$ atoms and $4 c$ of $\mathrm{Ni}$ atoms contain a certain number of vacancies (no atoms).

Thus, in the hypothetical compound $\mathrm{Lu}_{0.99} \mathrm{NiSb}$ the Fermi level $\varepsilon_{F}$ will change its position and is located at the edge of the valence band $\varepsilon v$ : the dielectric-metal conductivity transition will take place (Fig. 3), and free holes become the main current carriers. The location of the Fermi level $\varepsilon_{F}$ near the edge of the valence band or in the band itself is clear, because the absence of the $\mathrm{Lu}$ atom in the crystallographic position $4 a$ generates a structural defect of acceptor nature, which generates an acceptor level (band) in the bandgap. In this experiment we will have positive values of the thermopower coefficient $\alpha(T) \mathrm{Lu}_{0.99} \mathrm{NiSb}$, and the intersection of the Fermi level $\varepsilon_{F}$ and the valence band $\varepsilon_{V}$. will change the conductivity from the activation to the metallic one: the resistivity values $\rho(T)$ will increase with temperature. Such simulation results concerning the type of main electric current carriers correspond to the results of the experiment [1]. However, experimental studies [1] found that the temperature dependences of the resistivity $\rho(T)$ LuNiSb present high- and low-temperature activation regions, indicating the activation of holes from the Fermi level $\varepsilon_{F}$ to the edge of the valence band $\varepsilon_{V}$. Therefore, the Fermi level $\varepsilon_{F}$ in the real crystal is located in the bandgap of the semiconductor, and not the edge of the valence band $\varepsilon_{V}$, as shown by the simulation results for the case $\mathrm{Lu}_{0.99} \mathrm{NiSb}$.

The most acceptable results of experimental studies are the model of the electronic structure of the compound LuNiSb, which assumes the presence of vacancies in the crystallographic positions of $4 a \mathrm{Lu}$ atoms $(\sim 0.005)$ and $4 c \mathrm{Ni}$ atoms ( 0.04) (Fig. 3). In this model of the spatial arrangement of atoms and the presence of vacancies at positions $4 a$ and $4 c$, the $\mathrm{LuNiSb}$ compound is a hole-type semiconductor, in which the Fermi level $\varepsilon_{F}$ is located near the bandgap near the edge of the valence band $\varepsilon_{V}$. In such a model of the electronic structure of $p$-LuNiSb on the temperature dependences of the resistivity $\rho(T)$ LuNiSb will be present high- and low-temperature activation sites, and the value of the thermopower coefficient $\alpha(T)$, which corresponds to the results [1].

\section{Modeling of Kinetic Characteristics of $\mathrm{Lu}_{1-x} \mathrm{Zr}_{x} \mathrm{NiSb}$}

Modeling of the behavior of the resistivity $\rho(x, T)$ and the thermopower coefficient $\alpha(x, T) \mathrm{Lu}_{1-x} \mathrm{Zr}_{x} \mathrm{NiSb}$ was performed in the temperature range $T=4.2-450 \mathrm{~K}$. In Fig. 4 shows the results of modeling at temperatures $T=4.2-70 \mathrm{~K}$, and in Fig. $5-$ at temperatures $T=80-$ $450 \mathrm{~K}$. We can see that in LuNiSb the values of resistivity $\rho(x, T)$ in the temperature range $T=4.2-70 \mathrm{~K}$ decrease rapidly with increasing temperature (Fig. 4), which is characteristic of semiconductors when there is an increase in the number of free current carriers due to their activation from the Fermi level $\varepsilon_{F}$. Such carriers are free holes, which is indicated by positive values of the thermopower coefficient $\alpha(x, T)$. 

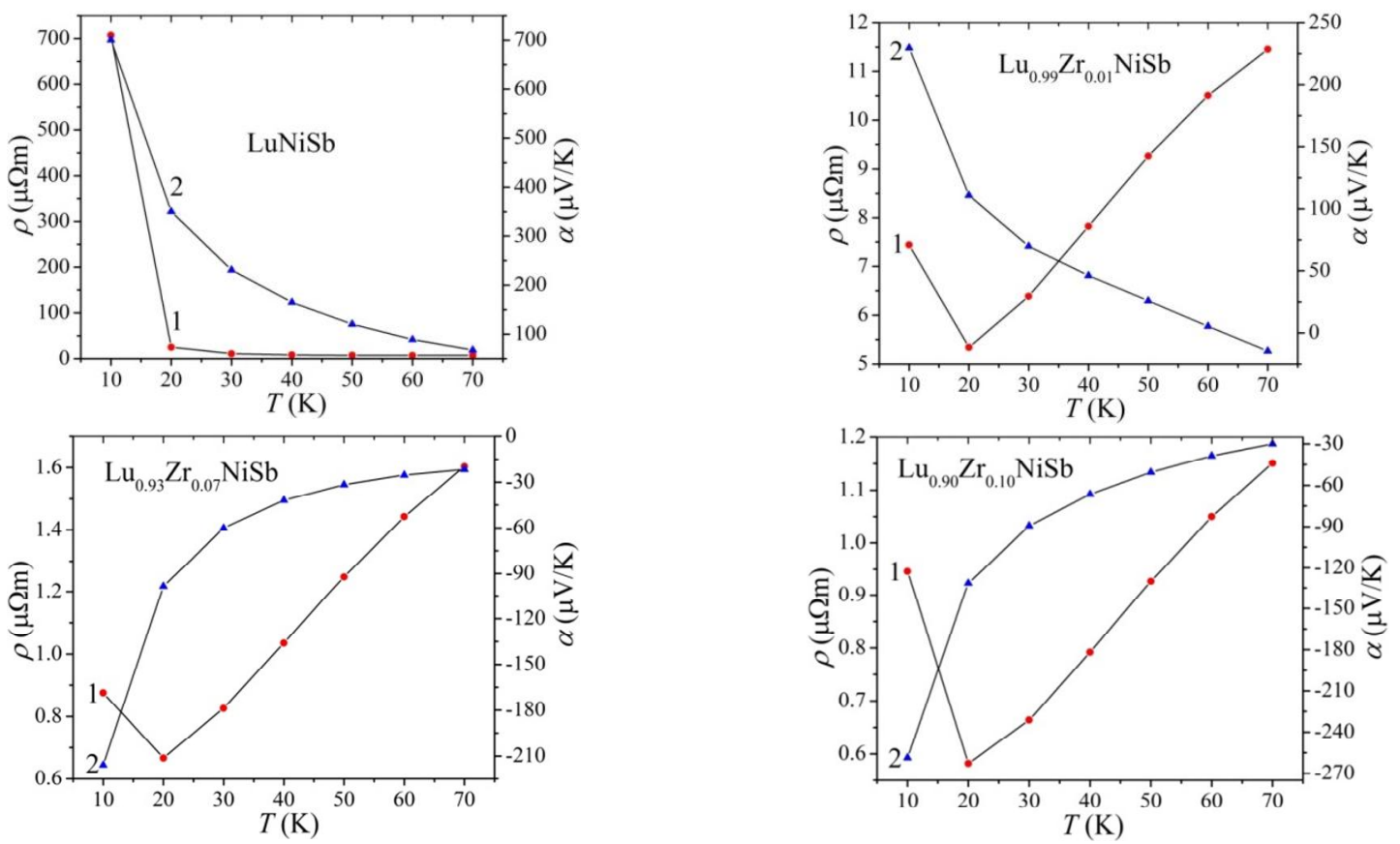

Fig. 4. Simulation of the change in the values of the resistivity $\rho(x, T)(1)$ and the thermopower coefficient $\alpha(x, T)(2)$ $L u_{1-x} Z r_{x} N i S b$ for temperatures $T=4.2-70 \mathrm{~K}$

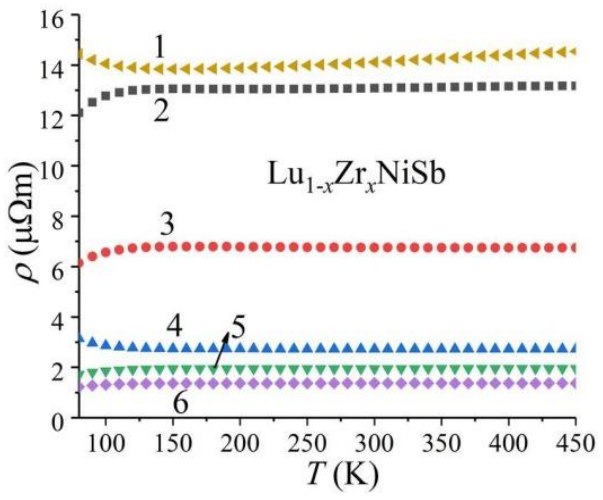

a

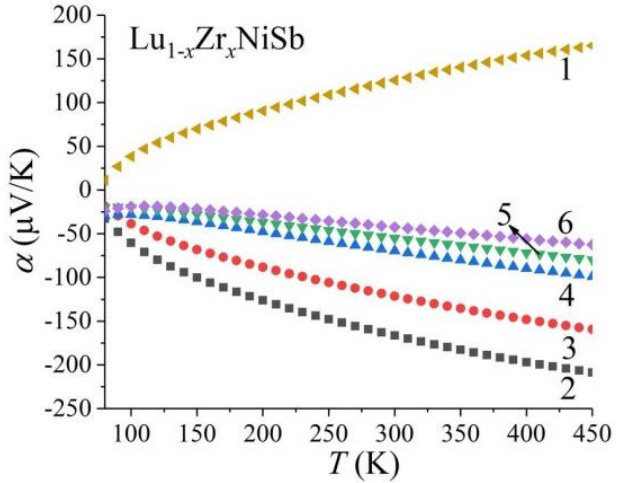

b

Fig. 5. Calculation of the change of the resistivity $\rho(T, x)(a)$ and the thermopower coefficient $\alpha(T, x)$ (b) $L u_{1-x} Z r_{x} N i S b$ for temperatures $T=70-450 \mathrm{~K}: 1-x=0 ; 2-x=0.01 ; 3-x=0.02 ; 4-x=0.05 ; 5-x=0.07 ; 6-x=0.10$

The fact that at all concentrations of $\mathrm{Zr}$ atoms in the temperature range $T=4.2-20 \mathrm{~K}$ the values of the resistivity $\rho(T, x) \quad \mathrm{Lu}_{1-x} \mathrm{Zr}_{x} \mathrm{NiSb}$ decrease indicates the depth of the formed donor zone. At low temperatures $(T<20 \mathrm{~K})$, the thermal energy is insufficient for the activation of electrons in the conduction band $\varepsilon_{C}$ $\mathrm{Lu}_{1-x} \mathrm{Zr}_{x} \mathrm{NiSb}$. However, at temperatures $T>20 \mathrm{~K}$ the concentration of free electrons becomes significant and the Fermi level $\varepsilon_{F}$ enters the conduction band $\varepsilon_{C}$ of the semiconductor, indicating the transition of the conductivity of the dielectric metal, which is the Mott transition [1].
Temperature dependences of specific electrical resistance $\rho(x, T)$ and thermopower coefficient $\alpha(x, T) \mathrm{Lu}_{1-}$ ${ }_{x} \mathrm{Zr}_{x} \mathrm{NiSb}, x>0$, in the temperature range $T=80-450 \mathrm{~K}$ do not contain special points, because the Fermi level $\varepsilon_{F}$ is located deep in the conduction band. This is evidenced by the metallic (non-activating) nature of the change in resistance values. Accordingly, the sign of the thermopower coefficient $\alpha(x, T)$ is also predicted to be negative, since electrons are the main carriers of electric current. In the case of LuNiSb, the values of the resistivity $\rho(T)$ and the thermopower coefficient $\alpha(T)$, as well as in the range of concentrations $T=4.2-70 \mathrm{~K}$, continue to decrease and increase, respectively. The holes remain the main carriers of current. 


\section{Conclusions}

Modeling of structural, thermodynamic, energy, and kinetic characteristics of thermosensitive material $\mathrm{Lu}_{1-x} \mathrm{Zr}_{x} \mathrm{NiSb}$ for the case of ordered crystal structure showed that in the semiconductor are generated as structural defects of donor nature, the concentration of which increases with increasing content of $\mathrm{Zr}$ atoms. The investigated solid solution $\mathrm{Lu}_{1-x} \mathrm{Zr}_{x} \mathrm{NiSb}$ is a promising thermometric material and requires further studies in the next works.

\section{Gratitude}

The authors express their gratitude to the members of the scientific seminar of the Department of Information and Measurement Technologies of Lviv Polytechnic National University for an interesting and meaningful discussion on the results of these studies.

\section{Conflict of interest}

The authors declare that there is no financial or other possible conflict related to this work.

\section{References}

[1] L. Romaka, Yu. Stadnyk, V.A. Romaka, P. Klyzub, V. Krayovskyy, V. Pashkevych, A. Horyn, P. Garanuk, "Synthesis and electrical transport properties of Er1-xScxNiSb semiconducting solid solution”,.Phys. Chem. Sol. St., no. 22(1), p.146-152, 2021. doi.org/10.15330/pcss.22.1.146-152

[2] M. Schruter, H. Ebert, H. Akai, P. Entel, E. Hoffmann, G.G. Reddy, "First-principles investigations of atomic disorder effects on magnetic and structural instabilities in transition-metal alloys", Phys. Rev. B, vol. 52, p.188-209, 1995.

[3] V. Moruzzi, J. Janak, A. Williams. Calculated Electronic Properties of Metals. NY, Pergamon Press, 1978.

[4] All-electron full-potential linearised augmented-plane wave (FP-LAPW) code. [Online]. Available: http://elk. sourceforge.net

[5] K. Momma, F. Izumi, "VESTA 3 for three-dimensional visualization of crystal, volumetric and morphology data", J. Appl. Crystallogr., vol. 41, p.653-658, 2008. dx.doi.org/ 10.1107/S0021889811038970

[6] I. Wolanska, K. Synoradzki, K. Ciesielski, K. Zaleski, P. Skokowski, D. Kaczorowski, Mater. Chem. Phys., vol.227, p.29-36, 2019. doi.org/10.1016/j.matchemphys.2019.01.056

[7] K. Synoradzki, K. Ciesielski, I. Veremchuk, H. Borrmann, P. Skokowski, D. Szymanski, Y. Grin, D. Kaczorowski, Materials, vol.12, p.1723-1730, 2019. doi:10.3390/ma12101723

[8] I. Karla, J. Pierre, R.V. Skolozdra, J. Alloys Compd., vol.265, p.42-50, 1998. doi.org/10.1016/S0925-8388(97)00419-2

[9] B. Shklovskii, A. Efros, Electronic Properties of Doped Semiconductors. Springer-Verlag, 1984. dx.doi.org/ 10.1007/978-3-662-02403-4 